\title{
Sensitivity of three serum antibody tests in a large outbreak of Legionnaires' disease in the Netherlands
}

Correspondence

Ed P. F. Yzerman

e.yzerman@streeklabhaarlem.nl

Received 11 October 2005

Accepted 26 January 2006

\author{
Ed P. F. Yzerman, ${ }^{1,2}$ Jeroen W. den Boer, ${ }^{3}$ Kamilla D. Lettinga, ${ }^{2}$ \\ Arnoud J. Schel, ${ }^{2}$ Joop Schellekens ${ }^{4}$ and Marcel Peeters ${ }^{5}$ \\ ${ }^{1}$ Regional Laboratory of Public Health Haarlem, Boerhaavelaan 26, 2035 RC Haarlem, \\ The Netherlands \\ ${ }^{2}$ Academic Medical Center, Amsterdam, The Netherlands \\ ${ }^{3}$ Municipal Health Service Kennemerland, Haarlem, The Netherlands \\ ${ }^{4}$ National Institute for Public Health and the Environment, Bilthoven, The Netherlands \\ ${ }^{5}$ Regional Laboratory of Public Health Tilburg, Tilburg, The Netherlands
}

\begin{abstract}
In 1999, an outbreak involving 188 patients with Legionnaires' disease (LD) occurred at a flower show in the Netherlands. This large outbreak provided the opportunity to evaluate serum antibody tests to assay anti-Legionella pneumophila, since limited data are available on the sensitivity of these tests. The sensitivities of an indirect serotype 1-6 immunofluorescence antibody test (IFAT), a rapid micro-agglutination test (RMAT) IgM serotype 1 antibody assay, and an ELISA to detect lgM and IgG serotype 1-7 antibodies, were evaluated using serum samples from LD patients related to the 1999 outbreak. Sensitivity was calculated using positive culture and/or a positive urinary antigen test as the gold standard in outbreak-related patients with radiographically confirmed pneumonia who fulfilled the epidemiological criteria. The IFAT, RMAT and ELISA showed sensitivities of 61,44 and $64 \%$, respectively. The sensitivity of the three tests combined was $67 \%$. In epidemic situations, however, high standing titres may be included in the laboratory evidence of LD cases. In the study population, high standing titres were found in $16 \%$ of cases. If the presence of high standing antibody titres was added to the criteria of a positive test, the sensitivities of IFAT, RMAT and ELISA were 86, 48 and $75 \%$, respectively. The sensitivity was $91 \%$ for all tests combined. The higher sensitivity for the combined use of tests is offset by a reduction in specificity to $97 \cdot 6 \%$. The results of this study indicate that using a combination of serologic tests in pneumonia patients suspected to have LD does not substantially improve sensitivity. The results suggest that in the microbiological diagnosis of LD, both IFAT and ELISA are reasonably sensitive assays. In an epidemic situation, both tests are highly sensitive, the IFAT more so than the ELISA.
\end{abstract}

\section{INTRODUCTION}

Legionnaires' disease (LD) is an acute pneumonia caused by Legionella spp., which are responsible for $2-5 \%$ of community-acquired pneumonias (CAPs) (Marston et al., 1997; Bohte et al., 1995). Patients are infected by inhalation of aerosols containing Legionella spp. or by aspiration of water contaminated with Legionella spp. Over $90 \%$ of LD cases are caused by Legionella pneumophila, of which $70-80 \%$ belong to serogroup type 1 (Yu et al., 2002;

Abbreviations: CAP, community-acquired pneumonia; EWGLI, European Working Group on Legionella Infections; IFAT, immunofluorescence antibody test; LD, Legionnaires' disease; RMAT, rapid micro-agglutination test.
Marston et al., 1997). Although over $90 \%$ of LD cases are sporadic, much can be learned from outbreak-related LD cases, including their microbiological diagnosis. Diagnosis of LD is mainly based on culture, antigen detection in urine, and antibody detection in serum. Culturing of Legionella spp. from respiratory secretions has a sensitivity ranging from 25 to $75 \%$ (Hoge \& Breiman, 1991). Urinary antigen tests, both enzyme immunoassay (EIA) and immunochromatographic assay (ICT) combine a $99 \%$ or higher specificity with a sensitivity ranging from 50 to $90 \%$ (Birtles et al., 1990; Dominguez et al., 1998, 1999; Ruf et al., 1990). Serological tests to identify antibodies to $L$. pneumophila were developed from 1977 onwards, starting with the indirect immunofluorescence antibody test (IFAT) (McDade et al., 1977). Several less laborious serological tests 
followed, among them numerous (mostly experimental) ELISAs and a micro-agglutination test (MAT). The first publication on an ELISA was in 1978 (Farshy et al., 1978). Although commercial ELISA kits have become widely used, few studies of their sensitivity and specificity have been published. The same holds for the MAT, which was also developed in 1978; this was followed by a rapid version, the rapid micro-agglutination test (RMAT), with identical test characteristics, in 1982 (Harrison \& Taylor, 1982). The sensitivity of all three assays has been tested using sporadic cases of LD and not in outbreak situations. A recent outbreak in the Netherlands (den Boer et al., 2002) provided a unique opportunity to evaluate the sensitivity of three serum antibody tests. This outbreak was caused by a $L$. pneumophila serogroup 1 strain. The nationwide alert for LD cases ensured optimal case finding, thereby decreasing patient selection bias. Furthermore, the outbreak situation allowed us to take the clinical spectrum into account, since all available clinical data for the LD patients related to this outbreak were recorded centrally. In addition, the conditions for a gold standard were favourable: two national reference laboratories performed all microbiological tests and a large collection of samples from a national serum bank was available to investigate the distribution of antibodies to L. pneumophila serogroup 1 among the Dutch population. To assess the sensitivity of the detection of serum Legionella antibodies, we performed three tests on serum specimens from outbreak-related LD cases: a serotype 1-6 antibody IFAT, an IgM serotype 1 antibody RMAT, and an ELISA to detect IgM and IgG serotype 1-7 antibodies (Boshuizen et al., 2003). The aim of our study was to compare the sensitivities of the three tests using confirmative and epidemic LD criteria, to see if combined use of the three tests would be justified, and to compare our results to published sensitivities of IFAT, RMAT and ELISA in both epidemic and non-epidemic settings.

\section{METHODS}

The outbreak. In February 1999, an outbreak involving 133 confirmed and 55 probable cases of $\mathrm{LD}$, according to the European Working Group on Legionella Infections (EWGLI) criteria (Anonymous, 1998), occurred in Bovenkarspel in the Netherlands. Of these patients, $89 \%$ were hospitalized. The outbreak investigation indicated that a whirlpool displayed at the consumer product division of an annual flower show was the most likely source of infection. Two L. pneumophila serogroup 1 strains were cultured from sputum of 29 patients. One of the strains was of the Allentown/France serogroup 1 subtype (Helbig et al., 2002) and was genotyped 010-London according to the EWGLI criteria (Fry et al., 2000). This strain was identified in 28 of the 29 culture-positive patients. Genotyping of the environmental L. pneumophila strains revealed that one L. pneumophila serogroup 1 strain found in the implicated whirlpool was of the 010-London genotype as well (Den Boer et al., 2002).

Patients. In this study, 133 patients with a confirmed Legionella pneumonia according to the EWGLI criteria were included after having obtained written consent from them or from their relatives. Of these patients, 104 fulfilled the criteria of the gold standard used in this study. The gold standard (identical to the EWGLI criteria, but excluding serological evidence of infection) was defined as a patient who had symptoms compatible with pneumonia, with radiological signs of infiltration, who had visited the site of the outbreak during the incubation period, and who had laboratory evidence of infection with $L$. pneumophila. Laboratory evidence included at least one of the following criteria:

(1) Isolation of L. pneumophila from a respiratory sample cultured on buffered charcoal yeast extract supplemented with 2-ketoglutarate $(\mathrm{BCYA}-\alpha)$, followed by genotyping and subsequent comparison to the environmental strain of the Bovenkarspel outbreak.

(2) Presence of L. pneumophila antigens in urine specimens, as shown in an antigen test. The three tests used in our study were two enzyme immunoassays, the Binax (Binax, Portland, ME, USA) and Biotest (Biotest AG, Dreiech, Germany) Legionella urinary antigen tests, and the BinaxNOW test, a qualitative immunochromatographic assay. All tests were used according to the manufacturers' instructions. Urine was concentrated and reread after $1 \mathrm{~h}$, as recommended (Dominguez et al., 1996; Helbig et al., 2001).

(3) All isolates from sputum samples were sent to the National Institute for Public Health and the Environment (RIVM) for genotyping of $L$. pneumophila. The Regional Laboratories of Public Health of Tilburg and Haarlem were the reference laboratories for the detection of $L$. pneumophila antigen in urine.

Classification of severity of disease. To evaluate exclusion bias by differences in severity of disease, the patients were divided into three clinical categories for CAP. Clinical data were collected from the hospital chart using a standardized case record form. Severity of pneumonia was scored on a five-point scale (0-4), according to the minor criteria for severity of CAP (Niederman et al., 1993), using the following clinical criteria at hospital admission: (1) respiratory frequency above 30 breaths per minute, (2) $\mathrm{PaO}_{2}$ below $60 \mathrm{~mm} \mathrm{Hg}$ or $\mathrm{O}_{2}$ saturation below $92 \%$, (3) bilateral or multilobar infiltration on chest X-ray, 4) systolic blood pressure below $90 \mathrm{~mm} \mathrm{Hg}$ or diastolic below $60 \mathrm{~mm} \mathrm{Hg}$.

Patients with a radiographically proven unilateral unilobar pneumonia, but without signs or symptoms according to the above-mentioned criteria, were classified as CAP category 1 (mild pneumonia). CAP category 2 consisted of patients who fulfilled only one of the criteria. According to our definition, these patients were suffering from a moderately severe pneumonia. CAP category 3 consisted of patients who presented themselves with two or more criteria and were considered to suffer from a severe pneumonia.

Serum samples and serum antibody tests. All 62 laboratories of clinical microbiology involved in diagnosis and treatment of the patients related to this outbreak sent all available serum samples to the Regional Laboratory of Public Health in Haarlem. After collection, serum samples were stored in portions at $-70{ }^{\circ} \mathrm{C}$.

The criteria for a paired serum sample confirmative for LD were defined as:

(1) A fourfold rise in IgG, IgM and/or IgA antibodies to L. pneumophila in paired acute-phase (0-15 days after disease onset) and convalescentphase sera, with final titre $\geqslant 1: 128$, using a commercial serotype 1-6 antibody IFAT (Polyvalent Legionella IFA Test Kit Serogroups 1-6, Meridian Bioscience Europe Srl.) (McDade et al., 1977).

(2) A fourfold rise in IgM antibodies to L. pneumophila in paired acutephase (0-15 days after disease onset) and convalescent-phase sera, with final titre $\geqslant 1: 32$ in accordance with the $99 \%$ cut-off value found in a serosurvey among healthy volunteers representative of the Dutch population (Boshuizen et al., 2003), using an IgM, serogroup 1 Philadelphia-I subtype, antibody RMAT (Farshy et al., 1978). 
(3) Seroconversion to positive IgM or IgG antibodies to L. pneumophila in paired acute-phase ( $0-15$ days after disease onset) and convalescentphase sera, in accordance with the age-specific $99 \%$ cut-off values found in a serosurvey among healthy volunteers representative of the Dutch population (Boshuizen et al., 2003), using a commercial ELISA to detect IgM and IgG serotype 1-7 antibodies (Serion classic ELISA, Institut Virion/Serion GmbH, Würzburg, Germany) (Etienne et al., 1999). The antigens in this test are prepared from cultures of different L. pneumophila serogroups and subtypes: serogroup 1 Knoxville and Philadelphia subtypes, serogroup 2 Togus-1 subtype, serogroup 3 Bloomington-2 subtype, serogroup 4 Los Angeles- 1 subtype, serogroup 5 Dallas-1 subtype, serogroup 6 Chicago-2 subtype, and serogroup 7 Chicago-8 subtype.

The criteria for a paired serum sample suggestive for LD used in an epidemic situation were defined as:

(1) High standing titres of IgG, IgM and/or IgA antibodies to $L$. pneumophila in paired acute-phase (0-15 days after disease onset) and convalescent-phase sera, with final titre $\geqslant 1: 128$ using a serotype 1-6 antibody IFAT, as described above.

(2) High standing titres of IgM antibodies to L. pneumophila in paired acute-phase (0-15 days after disease onset) and convalescent-phase sera, with final titre $\geqslant 1: 32$ using an IgM antibody RMAT, as described above.

(3) High standing titres of IgM or IgG antibodies to L. pneumophila in paired acute-phase (0-15 days after disease onset) and convalescentphase sera using an ELISA to detect IgM and IgG serotype 1-7 antibodies, as described above.

Sensitivity. Sensitivity was defined as the number of patients with a positive test result divided by the total number of patients tested. Sensitivity for confirmed LD was calculated using the confirmative criteria for LD. Sensitivity for an epidemic situation was calculated using the confirmative and the suggestive criteria for $\mathrm{LD}$.

Statistics. Statistical analysis was performed with the statistical program SPSS version 12.0 (Statistical Product and Service Solutions). Univariate analysis was used to identify factors potentially associated with test positivity: age, gender, clinical severity, number of days passed between onset of disease and collection of the first serum sample, and number of serum samples taken.

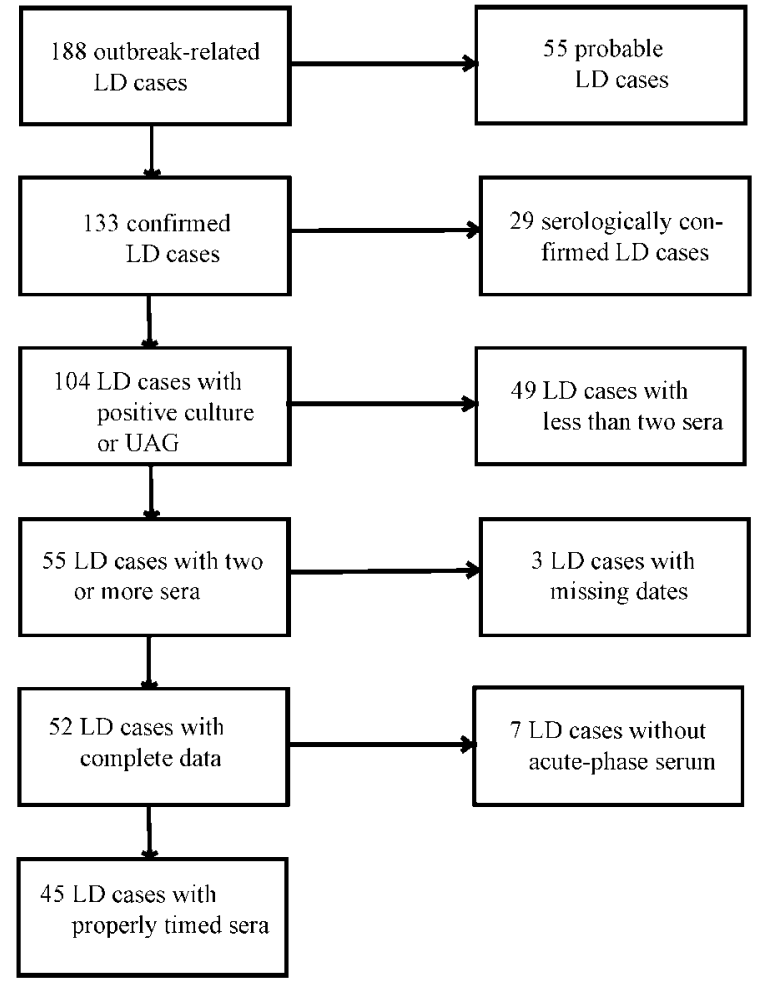

Fig. 1. Selection of LD patients in this study. UAG, urinary antigen.

\section{RESULTS AND DISCUSSION}

Two or more properly timed serum samples with complete sampling dates were available for evaluation from 45 of the 104 hospitalized patients who fulfilled the criteria of the gold standard used in this study (see Fig. 1 for patient selection). Table 1 shows that excluded patients died significantly more often from LD, although their CAP score

Table 1. Differences between 45 included and 59 excluded LD patients for factors possibly associated with exclusion bias

CAP category $1=$ mild pneumonia; CAP category $2=$ moderately severe pneumonia; CAP category $3=$ severe pneumonia.

\begin{tabular}{|lcc|}
\hline Factor & $\begin{array}{c}\text { Proportion of included } \\
\text { patients }\end{array}$ & $\begin{array}{c}\text { Proportion of excluded } \\
\text { patients }\end{array}$ \\
\hline Proportion of male gender & $60 \%$ & $68 \%$ \\
Mean age in years (range) & $63(21-78)$ & $65(25-84)$ \\
CAP category 1 & $27 \%$ & $26 \%$ \\
CAP category 2 & $32 \%$ & $31 \%$ \\
CAP category 3 & $41 \%$ & $43 \%$ \\
Admission to intensive care unit & $43 \%$ & $34 \%$ \\
Fatal outcome & $9 \%$ & $24 \% *$ \\
\hline
\end{tabular}

${ }^{\star}$ Difference is statistically significant. 
Table 2. Timing of convalescent serum sample(s) and presence of antibodies to L. pneumophila, as demonstrated by any of three assays, for 45 LD patients for whom two or more properly timed sera were available

\begin{tabular}{|lccc|}
\hline Time & $\begin{array}{c}\text { No. of patients } \\
\text { with available sera }\end{array}$ & Standing titre & $\begin{array}{c}\text { Fourfold rise in titre } \\
\text { or seroconversion }\end{array}$ \\
\hline 2-6 weeks & 37 & $6 / 37$ & $22 / 37$ \\
6-12 weeks & 12 & $0 / 12$ & $4 / 12$ \\
More than 12 weeks & 20 & $1 / 20$ & $4 / 20$ \\
\hline
\end{tabular}

${ }^{\star}$ Acute-phase serum was positive.

distribution was very similar to that of the included patients. Of the 45 included patients, 26 were men, with a mean age of 64 years (range 46-78 years) and 19 were women with a mean age of 60 years (range 21-77 years). The microbiological diagnosis was established by culture alone in one case, by culture and by urinary antigen test in nine cases and by urinary antigen test alone in 35 cases. The first serum to be tested for antibodies against $L$. pneumophila was collected $0-15$ days (mean 8 days) after the first day of signs and symptoms of pneumonia. From 22 patients two sera were available, from 13 patients three sera were available and from 10 patients four to eight sera were available for evaluation. Table 2 shows the timing of the convalescent sera. For one of the patients, the volume of serum was too small to perform all three tests, so only the RMAT test was performed. Patients with positive test results on average had their first serum taken 2 days earlier in the acute phase than patients with high standing titres: $7 \cdot 1$ versus $9 \cdot 4$ days after disease onset. This finding may suggest that in LD cases with high standing antibody titres, seroconversion had already occurred. In total, 7/45 (16\%) of patients showed high standing titres in all assays. Table 3 shows the sensitivities of the three tests and the sensitivity for the combination of all tests for both the non-epidemic and epidemic criteria. Evaluating the ELISA for its individual components, sera from $48 \%$ of the patients showed a seroconversion in IgM ELISA and $50 \%$ a seroconversion in IgG ELISA. Using the epidemic criteria, the sensitivities were 61 and $64 \%$, respectively.

It seems that the superior sensitivity of the IFAT and ELISA compared to the RMAT was mostly due to the identification of antibodies other than IgM. In this outbreak, the combination of tests was of limited value.

It has been suggested that in $\mathrm{LD}$, any diagnostic test with a specificity below $99 \%$ is unacceptable (Hoge \& Breiman, 1991). Most IFAT sensitivity studies use this criterion and show similar results in non-outbreak patient series. However, the sensitivity for the IFAT in the Philadelphia outbreak was $42 \%$ for a fourfold rise in titre, given a specificity of $99 \cdot 1 \%$. With high standing titres added to the criteria of an LD case, sensitivity was $68 \%$ (McDade et al., 1977). The IFAT used in our study seems more sensitive, probably due to the use of a polyvalent conjugate directed against IgG, IgM and IgA antibodies.

The sensitivity of the RMAT was $63 \%$ in a study evaluating sera from 119 patients with LD using a positive culture for $L$. pneumophila or its presence shown by direct fluorescence antibody as the gold standard (Harrison et al., 1987). The substantially higher sensitivity of the RMAT assay found in that study (63 versus $44 \%$ in our study) is difficult to explain. A different strain of L. pneumophila serogroup 1 was used, but the influence of different strains on RMAT

Table 3. Sensitivity of three serum L. pneumophila antibody tests using sera from 45 LD patients

\begin{tabular}{|lc|}
\hline Test criterion & Sensitivity \\
\hline Fourfold rise in IFAT & $61 \%(27 / 44)$ \\
Fourfold rise in RMAT & $44 \%(20 / 45)$ \\
Seroconversion in IgM or IgG ELISA & $64 \%(28 / 44)$ \\
Combination of the three tests above & $67 \%(30 / 45)$ \\
Fourfold rise or high standing titres in IFAT & $86 \%(38 / 44)$ \\
Fourfold rise or high standing titres in RMAT & $58 \%(26 / 45)$ \\
Seroconversion or high standing titres in IgM or IgG ELISA & $75 \%(33 / 44)$ \\
Combination of the three tests above & $91 \%(41 / 45)$ \\
\hline
\end{tabular}


sensitivity is unknown. There was a difference in the timing of the first serum sample (0-28 days after disease onset in the study of Harrison et al., 1987), but late sampling tends to underestimate the sensitivity. There were differences in study population: higher clinical severity, higher mortality, and over $50 \%$ immunocompromised patients in the study of Harrison et al. (1987).

To our knowledge, only two reports are available describing data from which an ELISA sensitivity based on a $99 \%$ or higher specificity could be inferred (Bangsborg et al., 1994; Wreghitt et al., 1982). Bangsborg et al. (1994) studied convalescent sera from 20 culture-proven LD patients and described an ELISA based on a lipopolysaccharide antigen with sensitivities of $70 \%$ for IgM and $75 \%$ for IgG, respectively. Although in the Bangsborg et al. (1994) study the sensitivity of an IgM and IgG ELISA combination is not described, the reported individual sensitivities are $10 \%$ higher than those found in our study. This may be due to a difference in the antigen used or a difference in study population. Wreghitt et al. (1982) studied sera from 11 patients and found sensitivities of 58 and $41 \%$ for IgM and IgG ELISA, respectively. In the Wreghitt et al. (1982) study, another serum antibody test was included in the gold standard, and no details were given concerning the timing of serum samples under study, complicating comparison to our data.

In conclusion, to diagnose L. pneumophila pneumonia, serology is still a valuable tool. This study is the first to compare the sensitivities of an IFAT, an RMAT and an ELISA in an outbreak situation. The IFAT and ELISA seem to yield similar results using confirmative LD criteria. In an epidemic situation, the IFAT seems superior. Combination of assays in an outbreak situation improves sensitivity only marginally, from 86 to $91 \%$. The data from this study suggest that early sampling may lead to a higher sensitivity for all tests.

\section{ACKNOWLEDGEMENTS}

We thank all hospital clinicians and microbiologists for requesting patients' permission and allowing us to collect clinical data and serum samples. Special thanks to Yvonne Boelens and Jacob P. Bruin, who performed the serum antibody tests.

\section{REFERENCES}

Anonymous (1998). Legionnaires' disease in Europe. Wkly Epidemiol Rec 73, 257-261.

Bangsborg, J. M., Shand, G. H., Hansen, K. \& Wright, J. B. (1994). Performance of four different indirect enzyme-linked immunosorbent assays (ELISAs) to detect specific IgG, IgA, and IgM in Legionnaires' disease. APMIS 102, 501-508.

Birtles, R. J., Harrison, T. G., Samuel, D. \& Taylor, A. G. (1990). Evaluation of urinary antigen ELISA for diagnosing Legionella pneumophila serogroup 1 infection. J Clin Pathol 43, 685-690.
Bohte, R., van Furth, R. \& van den Broek, P. J. (1995). Aetiology of community-acquired pneumonia: a prospective study among adults requiring admission to hospital. Thorax 50, 543-547.

Boshuizen, H. C., Den Boer, J. W., De Melker, H., Schellekens, J. F., Peeters, M. F., van Vliet, J. A. \& Conyn-van Spaendonck, M. A. (2003). Reference values for the SERION classic ELISA for detecting Legionella pneumophila antibodies. Eur J Clin Microbiol Infect Dis 22, 706-708.

Den Boer, J. W., Yzerman, E. P., Schellekens, J. \& 11 other authors. (2002). A large outbreak of Legionnaires' disease at a flower show, the Netherlands, 1999. Emerg Infect Dis 8, 37-43.

Dominguez, J. A., Manterola, J. M., Blavia, R. \& 7 other authors. (1996). Detection of Legionella pneumophila serogroup 1 antigen in nonconcentrated urine and urine concentrated by selective ultrafiltration. J Clin Microbiol 34, 2334-2336.

Dominguez, J. A., Gali, N., Pedroso, P., Fargas, A., Padilla. E., Manterola, J. M. \& Matas, L. (1998). Comparison of the Binax Legionella urinary antigen enzyme immunoassay, (EIA) with the Biotest Legionella Urin antigen EIA for detection of Legionella antigen in both concentrated and nonconcentrated urine samples. J Clin Microbiol 36, 2718-2722.

Dominguez, J., Gali, N., Matas, L., Pedroso, P., Hernandez, A., Padilla, E. \& Ausina, V. (1999). Evaluation of a rapid immunochromatographic assay for the detection of Legionella antigen in urine samples. Eur J Clin Microbiol Infect Dis 18, 896-898.

Etienne, J., Vandenesch, F. \& Lina, G. Jarraud (1999). Evaluation des SERION ELISA classic Legionella pneumophila 1-7 (IgG, IgM). Leusden, The Netherlands: Clindia Benelux bv.

Farshy, C. E., Klein, G. C. \& Feeley, J. C. (1978). Detection of antibodies to Legionnaires disease organism by microagglutination and micro-enzyme-linked immunosorbent assay tests. J Clin Microbiol 7, 327-331.

Fry, N. K., Bangsborg, J. M., Bernander, S. \& 11 other authors. (2000). Assessment of intercentre reproducibility and epidemiological concordance of Legionella pneumophila serogroup 1 genotyping by amplified fragment length polymorphism analysis. Eur J Clin Microbiol Infect Dis 19, 773-780.

Harrison, T. G. \& Taylor, A. G. (1982). A rapid microagglutination test for the diagnosis of Legionella pneumophila (serogroup 1) infection. J Clin Pathol 35, 1028-1031.

Harrison, T. G., Dournon, E. \& Taylor, A. G. (1987). Evaluation of sensitivity of two serological tests for diagnosing pneumonia caused by Legionella pneumophila serogroup 1. J Clin Pathol 40, 77-82.

Helbig, J. H., Uldum, S. A., Luck, P. C. \& Harrison, T. G. (2001). Detection of Legionella pneumophila antigen in urine samples by the BinaxNOW immunochromatographic assay and comparison with both Binax Legionella Urinary Enzyme Immunoassay, (EIA) and Biotest Legionella Urin Antigen, EIA. J Med Microbiol 50, 509-516.

Helbig, J. H., Bernander, S., Castellani, P. M. \& 13 other authors. (2002). Pan-European study on culture-proven Legionnaires' disease: distribution of Legionella pneumophila serogroups and monoclonal subgroups. Eur J Clin Microbiol Infect Dis 21, 710-716.

Hoge, C. W. \& Breiman, R. F. (1991). Advances in the epidemiology and control of Legionella infections. Epidemiol Rev 13, 329-340.

Marston, B. J., Plouffe, J. F., File, T. M., Jr, Hackman, B. A., Salstrom, S. J., Lipman, H. B., Kolczak, M. S. \& Breiman, R. F. (1997). Incidence of community-acquired pneumonia requiring hospitalization. Results of a population-based active surveillance study in Ohio. The Community-Based Pneumonia Incidence Study Group. Arch Intern Med 157, 1709-1718.

McDade, J. E., Shepard, C. C., Fraser, D. W., Tsai, T. R., Redus, M. A. \& Dowdle, W. R. (1977). Legionnaires' disease: isolation of a 
bacterium and demonstration of its role in other respiratory disease. N Engl J Med 297, 1197-1203.

Niederman, M. S., Bass, J. B., Jr, Campbell, G. D. \& 7 other authors.

(1993). Guidelines for the initial management of adults with community-acquired pneumonia: diagnosis, assessment of severity, and initial antimicrobial therapy, American Thoracic Society. Medical Section of the American Lung Association. Am Rev Respir Dis 148, 1418-1426.

Ruf, B., Schurmann, D., Horbach, I., Fehrenbach, F. J. \& Pohle, H. D. (1990). Prevalence and diagnosis of Legionella pneumonia: a, 3-year prospective study with emphasis on application of urinary antigen detection. J Infect Dis 162, 1341-1348.

Wreghitt, T. G., Nagington, J. \& Gray, J. (1982). An ELISA test for the detection of antibodies to Legionella pneumophila. J Clin Pathol 35, 657-660.

Yu, V. L., Plouffe, J. F., Pastoris, M. C. \& 8 other authors. (2002). Distribution of Legionella species and serogroups isolated by culture in patients with sporadic community-acquired legionellosis: an international collaborative survey. J Infect Dis 186, $127-128$. 